\title{
Droop Coefficient Design in Droop Control of Power Converters for Improved Load Sharing: An Artificial Neural Network Approach
}

\author{
Habibu Hussaini ${ }^{1}$, Tao Yang ${ }^{1 *}$, Yuan Gao ${ }^{1}$, Cheng Wang ${ }^{1}$, Tomislav Dragicevic ${ }^{2}$, Serhiy Bozhko ${ }^{1}$ \\ ${ }^{1}$ Power Electronics, Machines and Control (PEMC) Research Group, Faculty of Engineering, University of Nottingham, \\ Nottingham, NG7 2RD, UK \\ ${ }^{2}$ Center for Electric Power and Energy, Technical University of Denmark, Lyngby, Denmark \\ ${ }^{*}$ Corresponding Author: Tao.Yang@nottingham.ac.uk
}

\begin{abstract}
In this paper, a new approach for the design of the droop coefficient in the droop control of power converters using the artificial neural network (ANN) is proposed. In the first instance, a detailed more electric aircraft (MEA) electrical power system (EPS) circuit model is simulated in a loop using different combinations of the converters droop coefficients within a design space. The inaccurate output DC currents sharing of the converters due to the influence of the unequal cable resistance are then obtained from each of the simulations. The data generated is then used to train the NN to be a dedicated surrogate model of the detailed MEA EPS simulation. Thus, for any user-defined desired current sharing among the converters that are within the design space, the proposed $\mathrm{NN}$ can provide the optimal droop coefficients. This NN approach has been verified through simulations to ensure accurate current sharing between the converters as desired. Hence, can be used in the design of the droop coefficient to enhance the performance of the conventional droop control method.
\end{abstract}

Keywords-Artificial neural network, Droop coefficient, Cable resistance, More electric aircraft, Data generation

\section{INTRODUCTION}

More electric aircraft (MEA) is regarded as one of the major trends for future aircraft. One of the promising and potential DC electrical power system (EPS) architecture for the MEA application is as shown in Fig. $1[1,2]$. It consists of three parallel-connected generators, i.e., a low-pressure generator (LPG), a high-pressure Generator (HPG) and an auxiliary power unit, a common HVDC bus that is powered by the three parallel-connected generators and through which power is distributed in the system and loads. The LPG and the HPG are the main generators and are assumed to be permanent magnet synchronous generators (PMSGs)). The main generators obtain power from the aircraft engine through LP and HP shafts respectively as shown in Fig. 1. The APU provides power to the aircraft in an emergency. Pulse-width modulated active front-end controlled rectifier unit (AFE $\mathrm{CRU}$ ) are employed to control and regulate the output voltage of the three variable frequency generators as shown in Fig. 1. The loads are made up of resistive and constant power loads (CPLs). The MEA EPS can be regarded as a typical DC microgrid operating in the islanding mode.

The need to have the sources work together in a coordinated manner and share the load power demand accurately according to their respective power capacity especially under heavy load condition cannot be overemphasized. This is to ensure that the sources are not overloaded, thermally stressed and saturated [3]. Also, accurate load sharing among the sources could decrease the
DC bus voltage drop and improve the power quality of the MEA EPS.

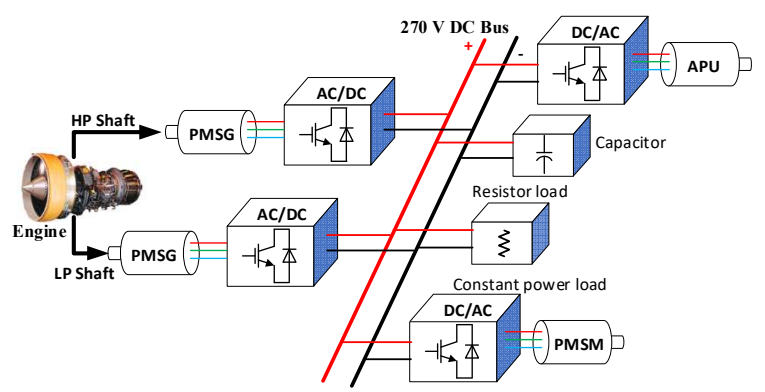

Fig. 1: A typical multi-source DC-grid in the MEA

Load sharing among the sources can be realized using the droop control method. However, the influence of unequal cable resistance on accurate load sharing is one of the limitations of the conventional droop control method. The error in load sharing becomes alarming when there is a huge variation between the corresponding subsystems cable resistances (i.e. huge variation in cable lengths) [3]. Generally, when high droop coefficients are set for the converters, this helps to achieve better load sharing performance but results in poor regulation of the DC bus voltage, particularly under heavy load condition. Conversely, when the droop coefficient set for the converters are small, the regulation of the DC bus voltage is enhanced while the load sharing accuracy is degraded [4]. Hence, there is a trade-off between the voltage regulation and accurate load sharing in the droop controlled DC microgrid. It can therefore be said that the choice of the droop coefficient plays a vital role in ensuring improve load sharing and DC bus voltage regulation in a system controlled using the droop control method.

A unified compensation framework that utilizes the common load condition to compensate for the load sharing errors is proposed in [5]. However, it is difficult to select the compensation coefficient. In [4], an enhanced droop control method (referred to as the line drop compensator) for accurate load sharing and voltage regulation is proposed for isolated and interconnected DC microgrids. However, the realization of accurate load sharing is entirely dependent on the accurate measurement of the corresponding subsystem cable resistance. The estimation of the cable resistance is prone to errors due to its variation with temperature. Hence, this approach has a practical limitation. Furthermore, any error in the measurement of the cable resistance will lead to an error in load power sharing and poor DC bus voltage regulation. Besides, it is difficult in practice to know the value of the corresponding subsystems cable resistance and most of the 
methods used to estimate the cable resistance require the use of many resources, time-consuming and are based on the injection of perturbations to the EPS. This has the potential of degrading the power quality of the EPS and increase the cost [6].

The conventional procedure used in power electronics system (PES) design is usually time-consuming and involves many iterative steps. Furthermore, in some cases, it depends on the experience of an expert and intuition without adequate data for reference. In this regard, the artificial intelligence (AI) method is here employed as a surrogate model to serve as a replacement of the original PES to reduce the design time [7, 8]. Therefore, this paper proposes a possible solution to the problem of inaccurate current sharing in the DC microgrid due to the influence of unequal line impedance by employing the artificial neural network (ANN) in the design of the droop coefficient in a fast and accurate manner for improved load sharing. By exploring all the combinations of the droop coefficients in the design space, the desired $\mathrm{NN}$ can be trained efficiently after data generation. Thereafter, the trained neural network is used to predict the optimal droop coefficients combination that will ensure an accurate/desired current sharing as defined by the user. Also, this approach has the advantage of not knowing the corresponding subsystem cable resistance. Since the method is implemented offline, it does not impose an additional computation burden on the system.

The rest of the paper is structured as follows. In section II the system architecture and its control model using the conventional voltage-mode droop control scheme are introduced. Section III discusses the proposed NN-based droop coefficient design approach for improved load sharing. The validation of the design approach is provided in Section IV. Section V concludes the paper.

\section{SYSTEM ARCHITECTURE}

A potential parallel-connected multi-source DC grid for the future MEA EPS architecture is shown in Fig. 2. This topology is made up of three sources $\left(G_{l-3}\right)$, three converters $\left(A R_{1-3}\right)$ and a CPL. The generators are assumed to be PMSGs. The main DC bus of the MEA EPS is $270 \mathrm{~V}$ and includes a capacitor bank $C_{b}$.

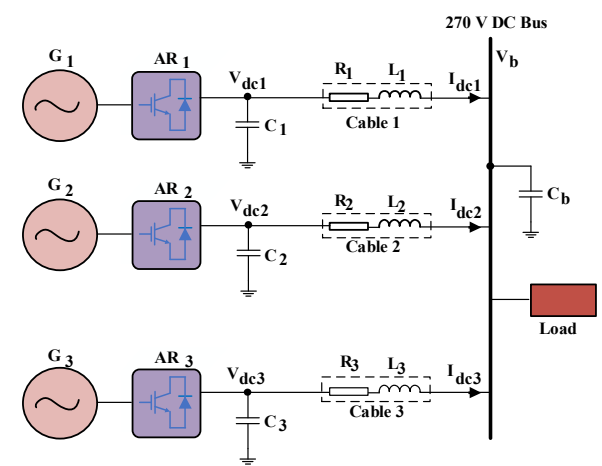

Fig. 2: A Potential Multi-source DC-grid for Future MEA EPS

The two-level voltage source converter (VSC) in Fig. 2 is controlled using the voltage-mode droop control scheme for current sharing among the converters in the MEA EPS as shown in Fig. 3. Only one source (i.e. PMSG) is shown in Fig. 3 to conserve space.

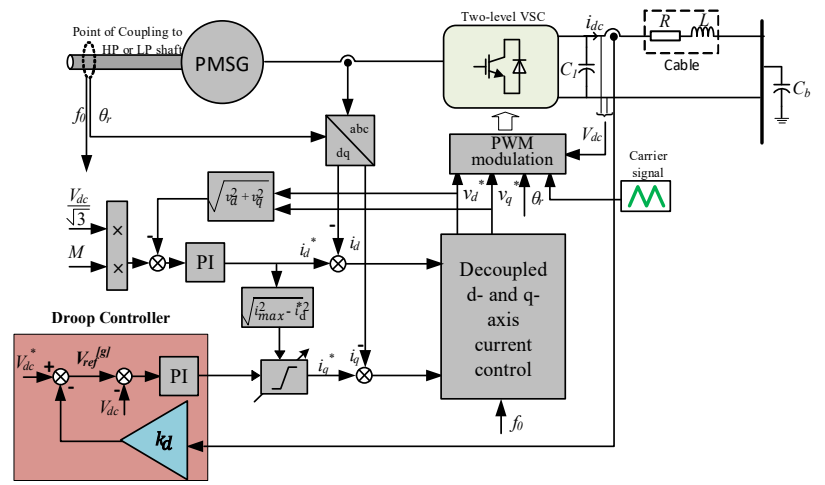

Fig. 3. Voltage-mode droop control scheme of a PMSG fed by an active rectifier (AR) in the MEA EPS

\section{A. Analysis of the Conventional Droop Control Method}

In the voltage-mode droop control scheme, the measured branch output DC current is used to generate the reference voltage, and this is expressed in (1).

$$
V_{d c i}{ }^{*}=V_{d c}^{*}-k_{d i} I_{d c i}
$$

where $i=1,2,3$ is the number of subsystems, $V_{d c}{ }^{*}$ is the rated DC bus voltage $(270 \mathrm{~V}), V_{d c i}{ }^{*}$ is the calculated reference voltage for each subsystem converter and $I_{d c i}$ is the output current of each converter in the system, $k_{d i}$ is the droop coefficient. The current sharing ratio among the sources (we are considering three sources) in steady-state is as expressed in (2), provided the effect of cable impedance on load sharing is ignored.

$$
I_{d c 1}: I_{d c 2}: I_{d c 3}=\frac{1}{k_{d 1}}: \frac{1}{k_{d 2}}: \frac{1}{k_{d 3}}
$$

where $k_{d i}$ are the coefficients of the droop gain. The droop coefficients are usually selected to be proportional to the generators ratings to ensure an accurate current sharing, based on the assumption that the same nominal voltage $V_{d c}{ }^{*}$ is applied to each of the droop characteristics.

When the voltage drop on the cable (in Fig. 3) is considered and the voltage control dynamics are neglected, the steady-state DC bus voltage can be expressed as in (3).

$$
V_{b}=V_{d c}^{*}-I_{d c i} R_{i}=V_{d c}^{*}-I_{d c i}\left(k_{d i}+R_{i}\right)
$$

where $V_{b}$ is the main DC voltage, $R_{i}$ is the resistance of the individual cable connecting the $i$ th source to the load. Hence, the current sharing among the sources, assuming they are supplying together can be expressed as in (4).

$$
I_{d c 1}: I_{d c 2}: I_{d c 3}=\frac{1}{k_{d 1}+R_{1}}: \frac{1}{k_{d 2}+R_{2}}: \frac{1}{k_{d 3}+R_{3}}
$$

It can be deduced from (4) that the cable resistance and droop gain will have an impact on the power sharing ratio of the sources in steady states. By increasing the droop gain or cable resistance, the power output of the sources will be decreased. Furthermore, when the droop gain and cable resistance are similar, the accuracy of the power sharing among the sources will be degraded due to the existence of the cable resistance. 
To achieve accurate load sharing, these two approaches are usually employed. The first approach is to increase the droop coefficient much higher than the cable resistance $\left(k_{d i} \gg R_{i}\right)$ such that the influence of the cable resistance on accurate load sharing becomes negligible. However, this will leads to poor voltage regulation and may affect the system's stability [5] and power quality [9]. Also, in low-voltage DC microgrids including the MEA EPS, the cable resistance cannot be simply ignored $[4,5]$. This is because the line impedance in the low voltage DC microgrid is predominantly resistive [10]. Moreover, the maximum droop gain that can be set is bounded by the maximum allowable DC bus voltage deviation and the full load current of the power converter [4] as expressed in (5).

$$
k_{d \max }=\frac{\Delta V_{b \max }}{i_{F}}
$$

where the power converter's full load current is represented as $i_{F}, k_{d \max }$ is the maximum allowable droop gain and $\Delta V_{b \max }$ is the maximum allowable deviation of the DC bus voltage. The second approach has to do with the compensation or modification of the droop gain according to the corresponding subsystem cable resistance as proposed in [4, 11] and referred to as the line drop compensator in [4]. In [4], the effective droop gain modification is realized through the addition of a compensation term to each of the subsystem (locally) using an active signal as shown in Fig. 4. This control method works in the same way as a virtual negative resistance to cancel the effect of the unequal cable resistance on accurate current sharing through compensation. Further details about the line drop compensator method can be found in [4]. However, since the method will be used to validate the proposed NN-based droop coefficient design, a brief mathematical analysis of the method will be provided.

\section{B. Line Drop Compensator Method}

When the influence of cable resistance is considered, this causes the effective droop gain to increase from $k_{d i}$ in (2) to $k_{d i}+R_{i}$ in (4), hence, leading to poor regulation of the DC bus voltage. On the other hand, accurate load sharing is not realized due to the unequal cable resistances connecting the parallel-connected DC sources to the load. The unequal cable impedance can be attributed to the difference in the relative distance (geographic location) between the DC sources and the load in the microgrid. Therefore, when the compensation term is added, and the voltage control dynamics are neglected, the new steady-state DC bus voltage is expressed in (6).

$$
V_{\text {bnew }}=V_{d c}^{*}-\left(k_{d i}+R_{i}\right) I_{d c i}+\left(R_{c o m p i} I_{d c i}\right)
$$

where $R_{\text {compi }}$ is the gain of the feedback proportional controller for the $i$ th controller as shown in Fig. 4. Therefore, from (6), the new or modified droop gain due to the compensation term $\left(R_{\text {compi }} I_{d c i}\right)$ and the new current sharing ratio for the voltage-mode droop control method is as expressed in (7) and (8) respectively.

$$
\begin{gathered}
k_{d i}^{n e w}=k_{d i}-R_{c o m p i} \\
I_{d c 1}^{\text {new }}: I_{d c 2}^{\text {new }}: I_{d c 3}^{\text {new }}=\frac{1}{k_{d 1}^{\text {new }}+R_{1}}: \frac{1}{k_{d 2}^{\text {new }+R_{2}}}: \frac{1}{k_{d 3}^{\text {new }}+R_{3}}(8) \\
R_{\text {compi }}=R_{i}
\end{gathered}
$$

where $k_{\text {dinew }}$ is the modified (new) droop gain due to the introduction of the compensation term. Fig. 4 shows the control block diagram of the implementation of the line drop compensator in the MEA EPS for the voltage-mode droop control scheme.

From (9), it can be observed that the gain of the proportional controller in each subsystem must be set equal to the estimated cable resistance of the corresponding subsystem to achieve accurate load sharing. Since accurate load sharing is dependent on accurate estimation of the cable resistance, an error in the cable resistance estimation will lead to an error in load sharing [2]. To find a solution to this problem, an intelligent approach that requires no knowledge of the corresponding subsystem cable resistance is proposed and because results can be obtained accurately and very fast.

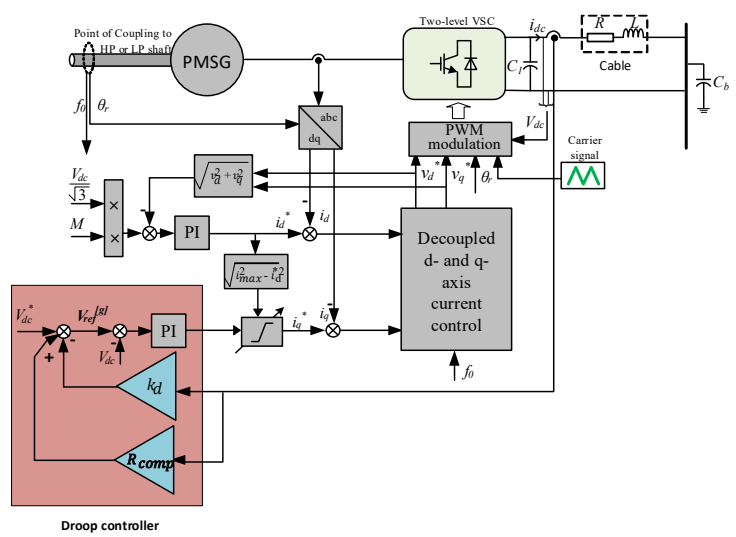

Fig. 4. Voltage-mode Line Drop Compensation Droop Control Scheme of a PMSG fed by an Active Rectifier in the MEA EPS

\section{PROPOSED ANN-BASED Droop COEFFICIENT DESIGN APPROACH}

\section{A. The methodology of the Proposed Approach}

Three steps are involved in the design of the droop coefficient for improved current sharing. A flow chart showing the design steps to be followed in the NN-based droop coefficient design approach is shown in Fig. 5.

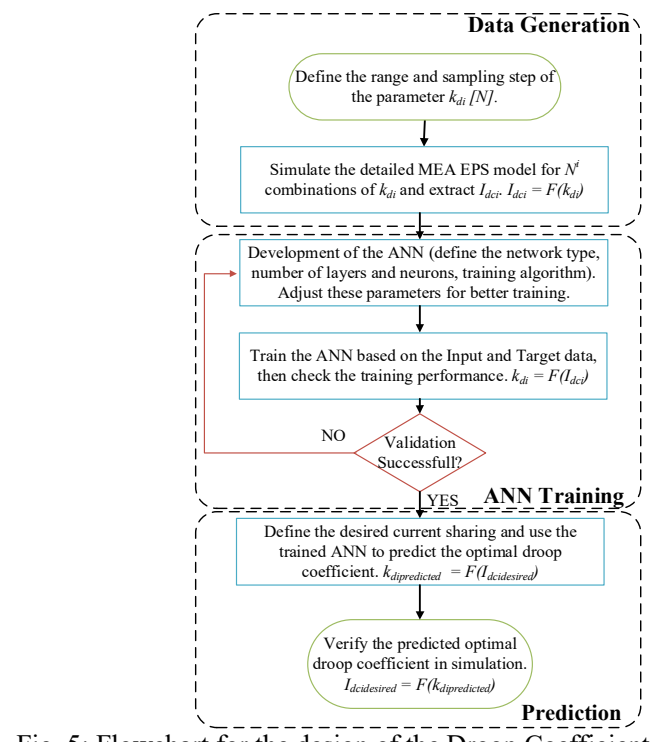

Fig. 5: Flowchart for the design of the Droop Coefficient using the ANN approach

The first step involves the collection of data from a detailed simulation of the MEA EPS model shown in Fig. 3 for every combination of the design parameter. The data 
obtained is then used to train the NN to become a surrogate model of the MEA EPS model in the second step. After training, based on a user-defined desired current sharing, the optimal design parameter (in this paper, the droop coefficient) that will yield the desired current sharing can be predicted by the trained NN in a fast and accurate manner. It is noteworthy to mention that the data generation and training of the $\mathrm{NN}$ steps need to be carried out only once for the detailed system model parameters.

\section{B. Procedure for Data Generation}

The detailed MEA EPS system control model which is used as the case study in this paper (shown in Fig. 3) was developed using the MATLAB SIMULINK(C) The simulations are carried out using three sources $\left(G_{1-3}\right)$ as shown in Fig. 2. A CPL of $40 \mathrm{~kW}$ was applied to the MEA EPS at $0.04 \mathrm{~s}$ during data generation. The simulation was set to run for $0.06 \mathrm{~s}$. The MEA EPS and equivalent DC cable parameters used in the simulation are as shown in TABLE I and II respectively.

TABLE I. ElECTRICAL POWER SySTEM PARAMETERS

\begin{tabular}{lcc}
\hline \hline Parameter & Symbol & Value \\
\hline \hline Rated Voltage of main DC Bus & $V_{d c}^{*}$ & $270 \mathrm{~V}$ \\
Local Shunt Capacitor & $C_{i}$ & $1.2 \mathrm{mF}$ \\
Main DC bus capacitor & $C_{b}$ & $0.6 \mathrm{mF}$ \\
Converter 1 Droop gain & $k_{d 1}$ & $1 / 4.250$ \\
Converter 2 Droop gain & $k_{d 2}$ & $1 / 4.250$ \\
Converter 3 Droop gain & $k_{d 3}$ & $1 / 4.250$ \\
\hline \hline
\end{tabular}

TABLE II. EQUIVALENT DC CABLES PARAMETERS

\begin{tabular}{cccc}
\hline \hline & $\begin{array}{c}\text { Resistance }\left(R_{i}\right) \\
(0.6 \mathrm{~m} \Omega / \mathrm{m})\end{array}$ & $\begin{array}{c}\text { Inductance }\left(L_{i}\right) \\
-(0.2 \mu \mathrm{H} / \mathrm{m})\end{array}$ & Length $(\mathrm{m})$ \\
\cline { 2 - 4 } Cable 1 & $3 \mathrm{~m} \Omega$ & $1 \mu \mathrm{H}$ & 5 \\
\hline Cable 2 & $30 \mathrm{~m} \Omega$ & $10 \mu \mathrm{H}$ & 50 \\
\hline Cable 3 & $15 \mathrm{~m} \Omega$ & $5 \mu \mathrm{H}$ & 25 \\
\hline \hline
\end{tabular}

The sweeping range and sampling step of the droop coefficient $\left(k_{d 1}, k_{d 2}, k_{d 3}\right)$ used in the simulation are presented in TABLE III. The sweep range is selected based on the converters conventional droop gain settings for the desired current sharing ratio $(1: 1: 1)$ shown in TABLE I. The droop coefficient sweep range is selected to cover $\pm 10 \%$ of the conventional droop coefficients to ensure a feasible design space with high fidelity.

TABLE III. DESIGN OF THE SweEP VALUES

\begin{tabular}{|c|c|c|c|}
\hline Variable & Range & $\begin{array}{c}\text { Sampling } \\
\text { Step }\end{array}$ & $\begin{array}{c}\text { Number of } \\
\text { Samples }\end{array}$ \\
\hline$k_{d 1}$ & {$[1 / 3.8251 / 4.675]$} & 0.085 & $11 \times 11 \mathrm{x}$ \\
\hline$k_{d 2}$ & {$[1 / 3.8251 / 4.675]$} & 0.085 & $11=1331$ \\
\hline$k_{d 3}$ & {$[1 / 3.8251 / 4.675]$} & 0.085 & \\
\hline
\end{tabular}

It can be observed from TABLE III that 11 settings for each of the design variable were tested, thereby making a total of 1331 combinations of the droop coefficient. Multiple simulations are carried out for every combination of the droop coefficients $k_{d i}\left(k_{d l}, k_{d 2}, k_{d 3}\right)$ and the output DC current $I_{d c i}\left(I_{d c l}\right.$, $\left.I_{d c 2}, I_{d c 3}\right)$ of the converters are obtained and recorded from each of the simulations. It is important to mention that the output DC currents of the converters are not in the desired sharing ratio $(1: 1: 1)$ due to the influence of the cable resistance. The multiple simulations were carried out in a loop and the process was automated with the aid of MATLAB codes developed and run from a MATLAB script file. Furthermore, the simulations were carried out on a standard personal computer with a quadcore processor. The simulations result were obtained within around 4 hours. The data generated from the simulations are used as the input-target sample data used to train the NN. At this data generation step, the relationship between the droop coefficients and the output DC currents from the converters can be represented as in (10).

$$
y=F 1(x) \leftrightarrow\left(I_{d c 1}, I_{d c 2}, I_{d c 3}\right)=F 1\left(k_{d 1}, k_{d 2}, k_{d 3}\right)
$$

where $k_{d 1}, k_{d 2}$, and $k_{d 3}$ are the droop coefficients combinations within the design space used for the multiple simulations and $I_{d c 1}, I_{d c 2}$, and $I_{d c 3}$ are the output of the converter DC currents obtained from each of the simulations.

\section{Structure and Training of the ANN}

In this paper, the feedforward neural network (FFNN) structure is selected to train the $\mathrm{NN}$ due to the static relationship between the input and output data [12]. Users do not need to specify the functional relationship between the model inputs-outputs because the powerful FFNN will learn it automatically by using only several internal training parameters (i.e. weights and bias). A detailed description of the FFNN can be found in [13]. A very important factor in the training of the neural network is the selection of the optimal number of neurons. The neurons present in each layer process the information they received from the layer before them. In this paper, the algorithm used in training the NN (LevenbergMarquardt method) and the training process are implemented in MATLAB's Neural Network Toolbox.

The NN structure is made up of three layers with 3 neurons in the input layer, 11 neurons in the hidden layer and 3 neurons in the output layer as shown in Fig. 6. At this training stage, the relationship between the droop coefficients and the output DC current shared among the converters is represented as in (11).

$$
y=F 2(x) \leftrightarrow\left(k_{d 1}, k_{d 2}, k_{d 3}\right)=F 2\left(I_{d c 1}, I_{d c 2}, I_{d c 3}\right)
$$

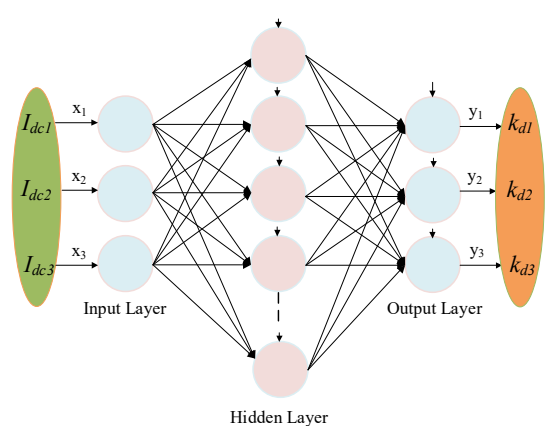

Fig. 6: Structure of the three-layer FFNN which serves as a surrogate model of the MEA EPS model shown in Fig. 3. The internal weights and bias terms are not included for simplicity.

It is important to mention that the choice of the number of neurons used in the hidden layer(s) to train the $\mathrm{NN}$ has a significant impact on the performance of the NN (overfitting or underfitting). As a rule of thumb, it is recommended that one starts with a relatively small number of neurons and then increase it gradually based on the observed training error [7]. This is a trial-and-error process and can be carried out very fast since the training can be completed within a few seconds $[14,15]$. In this paper and for the three sources MEA EPS used as a case study, the 11 neurons selected in the hidden layer of the FFNN structure used for training provides a very good match between the droop coefficient combinations used as 
input to the detailed simulation model and the $\mathrm{NN}$ model prediction as shown in Fig. 7. Furthermore, the root mean squared error (RMSE) is used to validate the performance of the NN training. The RMSE is the absolute difference between the output of the trained ANN and the targeted data used in training. The closer the RMSE value to zero, the better the training of the NN (its predictive capability). TABLE IV shows the calculated RMSE. Therefore, this shows that the neural network is well trained.

TABLE IV. NN PREDICTION ERROR OF THE TRAINING DATA

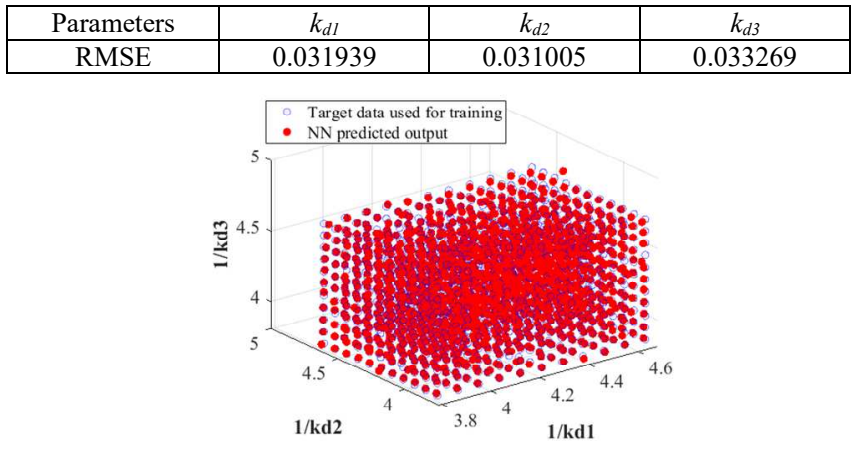

Fig. 7. Comparison between the droop coefficient combinations used as input to the detailed MEA EPS model and the NN model prediction

The NN was trained with the output DC currents of the converters recorded from each of the multiple simulations as inputs and the droop coefficient combinations as the target outputs. After training, the trained NN can be used to predict the optimal droop coefficient combination that will yield the desired accurate current sharing. The predicted optimal droop coefficient combination can then be used to share the load power demand among the generators as desired (defined by the user) using the conventional droop control method.

Therefore, after training, the relationship between the predicted optimal droop coefficients and the user-defined desired output DC current sharing is represented in (12).

$$
\begin{aligned}
y= & F 2(x) \leftrightarrow\left(k_{d 1}^{\text {pred }}, k_{d 2}^{\text {pred }}, k_{d 3}^{\text {pred }}\right)= \\
& F 2\left(I_{d c 1}^{\text {desired }}, I_{d c 2}^{\text {desired }}, I_{d c 3}^{\text {desired }}\right)(12)
\end{aligned}
$$

where $k_{d 1}^{\text {pred }}, k_{d 2}^{\text {pred }}, k_{d 1}^{\text {pred }}$, and $k_{d 3}^{\text {pred }}$ are the optimal droop coefficient combinations predicted by the trained $\mathrm{NN}$ based on the user-defined desired output DC current sharing among the converters $\left(I_{d c 1}^{\text {desired }}, I_{d c 2}^{\text {desired }}, I_{d c 3}^{\text {desired }}\right)$.

\section{APPLICATION AND VALIDATION OF PROPOSED THE DESIGN APPROACH IN THE MEA EPS}

Before applying the neural network in the MEA EPS for the prediction of the optimal droop coefficient, there is a need to first quantify a desired current sharing. This can be easily realized following the steps in the flow chart in Fig. 8. The general idea is first to find the feasible design space (3D intersection) of $I_{d c 1}, I_{d c 2}$, and $I_{d c 3}$, then chose the centre value as the $I_{d c i}$ desired.

The following desired output DC current sharing were obtained from the quantification process in Fig. 8, $I_{d c l}=51.82$ (A), $I_{d c 2}=51.82(\mathrm{~A})$ and $I_{d c 3}=51.82(\mathrm{~A})$ and the predicted droop coefficient combinations by the NN that will yield the desired current sharing are $k_{d l}{ }^{\text {pred }}=1 / 4.1357, k_{d 2}{ }^{\text {pred }}=1 / 4.6540$ and $k_{d 3}{ }^{\text {pred }}=1 / 4.3495$. This has been validated through simulation. However, since the line drop compensator will be used to validate the proposed method, the current sharing (i.e $I_{d c 1}=51.71(\mathrm{~A}), I_{d c 2}=51.71(\mathrm{~A})$ and $I_{d c 3}=51.71(\mathrm{~A})$ presented in TABLE $\mathrm{V}$ ) obtained from the line drop compensator is used as input to the trained $\mathrm{NN}$.

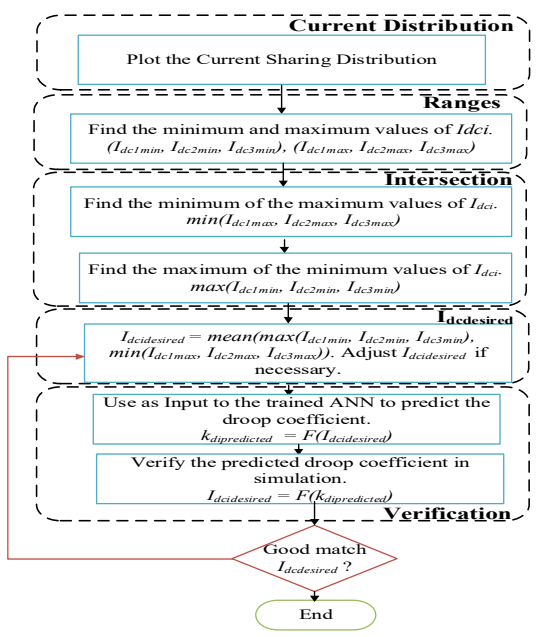

Fig. 8: Quantifying the Desired Current Sharing

The trained $\mathrm{NN}$ is then used to predict the optimal droop coefficients combination that will ensure these desired output DC currents sharing among the converters. The predicted optimal droop coefficients by the NN are $k_{d l}$ pred $=1 / 4.3099$, $k_{d 2^{p e d}}=1 / 4.8705$ and $k_{d 3^{p r e d}}=1 / 4.5387$. The compensated droop coefficients obtained using the line drop compensator are $k_{d 1}{ }^{\text {new }}=1 / 4.3049, k_{d 2}{ }^{\text {new }}=1 / 4.8711$ and $k_{d 3}{ }^{\text {new }}=1 / 4.5394$. The approximate values of these droop coefficients are as presented in TABLE V. Thus, for any user-defined current sharing that is within the feasible designed space of the droop coefficients used in training the NN, the NN can predict the optimal droop coefficients that will yield the desired current sharing.

The performance of the proposed NN-based droop coefficient design approach is compared to the line drop compensator droop control method in [4] and the conventional droop control method through simulation in MATLAB SIMULINK. The simulation parameters for the comparison are the same as those shown in TABLE I and TABLE II. A CPL of $20 \mathrm{~kW}$ was applied to the detailed MEA EPS simulation model at $0.04 \mathrm{~s}$ and increased by steps of $+10 \mathrm{~kW}$ at $0.05 \mathrm{~s}$, and $0.054 \mathrm{~s}$ during the simulation. The simulation results obtained for the current/power sharing between the three generators using the three methods are as summarized in TABLE V. Fig. 9 (a), (b) and (c) also show the simulation results for the output DC currents sharing when the three methods are implemented. Fig. 9 (d) shows the comparison of the DC bus voltage regulation when the three methods are implemented.

It can be seen from TABLE $V$ that the current sharing ratio among the three converters in steady-state using the conventional droop control methods is 1:0.898:0.952. The inaccurate current sharing in the conventional droop control methods is due to the influence of the cable resistance. On the other hand, the line drop compensation droop control method and the proposed NN-based droop coefficient design approach can achieve the desired sharing ratio of 1:1:1 as shown in TABLE V. The little error in the proposed method could be attributed to the choice of the number of neurons in the hidden layer. The proposed method has a better performance when compared to the conventional droop control method; 
moreover, it is also able to achieve accurate current sharing without knowing the corresponding subsystem cable resistance, unlike the line drop compensator droop control method. This priority of $\mathrm{NN}$ is the main motivation of this study, which can quickly find the desired droop coefficients for load sharing based on the sample data collected from a simulation loop, i.e. with no need for detailed information and substantial derivations.

TABLE V. Summary OF Simulation RESUlts AT $0.054 \mathrm{~S}$

\begin{tabular}{|c|c|c|c|c|c|c|}
\hline \multirow{2}{*}{$\begin{array}{l}\text { Conve } \\
\text { ntional }\end{array}$} & $k_{d l}$ & $k_{d 2}$ & $k_{d 3}$ & $I_{d c I}$ & $I_{d c 2}$ & $I_{d c 3}$ \\
\hline & $1 / 4.25$ & $1 / 4.25$ & $1 / 4.25$ & 54.61 & 49.05 & 51.99 \\
\hline \multirow{2}{*}{$\begin{array}{l}\text { Line } \\
\text { Drop }\end{array}$} & $k_{d l}{ }^{\text {new }}$ & $k_{d 2}{ }^{\text {new }}$ & $k_{d 3^{n e w}}$ & $I_{d c l}{ }^{\text {new }}$ & $I_{d c 2^{n e w}}$ & $I_{d c 3^{n e w}}$ \\
\hline & $1 / 4.30$ & $1 / 4.87$ & $1 / 4.54$ & 51.71 & 51.71 & 51.71 \\
\hline \multirow[t]{2}{*}{ ANN } & $k_{d I}$ pred & $k_{d 2}{ }^{\text {pred }}$ & $k_{d 3^{\text {pred }}}$ & $I_{d c I}$ & $I_{d c 2}$ & $I_{d c 3}$ \\
\hline & $1 / 4.30$ & $1 / 4.87$ & $1 / 4.54$ & 51.74 & 51.70 & 51.70 \\
\hline
\end{tabular}

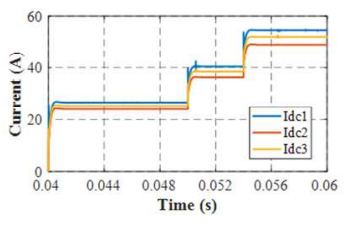

(a)

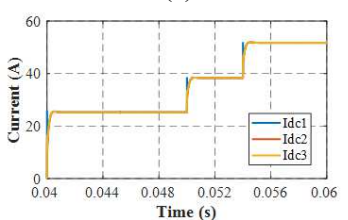

(c)

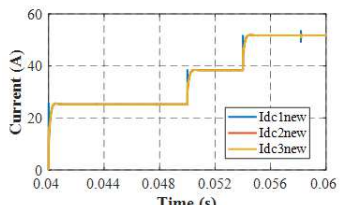

(b)

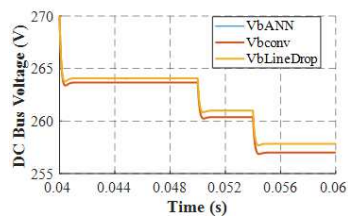

(d)
Fig. 9: Simulation Results for Desired Current Sharing Ratio (1:1:1) (a) Conventional Droop Control Method (b) Line Drop Compensation Droop Control Method and (c) Proposed ANN-based Design Approach (d) DC bus voltage regulation

Furthermore, in the three methods, the DC bus voltage decreases as the load current increases. When a constant power load of $40 \mathrm{~kW}$ was applied to the system at $0.054 \mathrm{~s}$, the main DC bus voltage dropped to $257 \mathrm{~V}\left(V_{\text {bconv }}\right)$ and $257.8 \mathrm{~V}$ ( $V_{\text {LineDrop }}$ and $\left.V_{b A N N}\right)$ from its initial value of $270 \mathrm{~V}$ due to the increase in the load current for all the three methods, as shown in Fig. 9 (d). However, both the line drop compensation droop control method and the proposed NN-based droop coefficient design approach can improve the DC bus voltage regulation due to the compensation of the cable impedance.

\section{CONCLUSION}

A new artificial neural network-based approach in the design of the droop coefficient for improved load sharing in the droop control of power converters is presented. After training, the NN can be used to predict the optimum droop coefficients combinations for any user-defined converter output DC currents sharing within the design space in a fast and accurate manner. The approach can be applied to the DC microgrid for improved load sharing irrespective of the variation in the geographic location of the sources (cable lengths) from the load. In the case study, the proposed NN approach is validated in simulation. Based on that, it is discussed and compared to the other two commonly used methods at the end.

\section{ACKNOWLEDGMENT}

This project has received funding from the Clean Sky 2 Joint Undertaking under the European Union's Horizon 2020 research and innovation programme under grant agreement No 807081. The author Habibu Hussaini extends his profound appreciation to the Petroleum Technology Development Fund (PTDF), Nigeria, for the scholarship funding.

\section{REFERENCES}

[1] J. Chen, C. Wang and J. Chen, "Investigation on the selection of electric power system architecture for future more electric aircraft," IEEE Transactions on Transportation Electrification, vol. 4, no. 2, pp. 563576, 2018.

[2] F. Gao, S. Bozhko, G. Asher and P. Wheeler, "Comparative Study of Power Sharing Strategies for the DC Electrical Power System in the MEA," in SAE Technical Paper (No. 2015-01-2410), 2015.

[3] F. Chen, R. Burgos, D. Boroyevich, J. C. Vasquez and J. M. Guerrero, "Investigation of nonlinear droop control in DC power distribution systems: Load sharing, voltage regulation, efficiency, and stability," IEEE Transactions on Power Electronics, vol. 34, no. 10, pp. 94049421, 2019.

[4] A. Tah and D. Das, " An enhanced droop control method for accurate load sharing and voltage improvement of isolated and interconnected DC microgrids," IEEE Transactions on Sustainable Energy, vol. 7, no. 3, pp. 1194-1204, 2016.

[5] N. Yang, D. Paire, F. Gao, A. Miraoui and W. Liu, "Compensation of droop control using common load condition in DC microgrids to improve voltage regulation and load sharing," International Journal of Electrical Power \& Energy Systems, vol. 64, pp. 752-760, 2015.

[6] H. Gu, X. Guo, D. Wang and W. Wu, "Real-time grid impedance estimation technique for grid-connected power converters," in 2012 IEEE International Symposium on Industrial Electronics, May 2012, pp. 1621-1626.

[7] S. Zhao, F. Blaabjerg and H. Wang, "An overview of artificial intelligence applications for power electronics," IEEE Transactions on Power Electronics, vol. 36, no. 4, pp. 4633 - 4658, 2021.

[8] T. Dragičević, P. Wheeler and F. Blaabjerg, "Artificial intelligence aided automated design for reliability of power electronic systems," IEEE Transactions on Power Electronics, vol. 38, no. 4, pp. 71617171, August 2019.

[9] F. Gao, S. Bozhko, G. Asher, P. Wheeler and C. Patel, "An Improved Voltage Compensation Approach in a Droop-Controlled DC Power System for the More Electric Aircraft," IEEE Transactions on Power Electronics, vol. 31, no. 10, pp. 7369 - 7383, 2016.

[10] Z. Shuai, D. He, J. Fang, Z. J. Shen, C. Tu and J. Wang, "Robust droop control of DC distribution networks," IET Renewable Power Generation, vol. 10, no. 6, pp. 807-814, 2016.

[11] C. Liu, J. Zhao, S. Wang, W. Lu and K. Qu, "Active identification method for line resistance in DC microgrid based on single pulse injection," IEEE Transactions on Power Electronics, vol. 33, no. 7, pp. 5561-5564, July 2018

[12] M. Novak, T. Dragicevic and F. Blaabjerg, "Weighting factor design based on Artificial Neural Network for Finite Set MPC operated 3LNPC converter," in 2019 IEEE Applied Power Electronics Conference and Exposition (APEC), Anaheim, CA, USA, March 2019, (pp. 77-82).

[13] B. K. Bose, "Neural network applications in power electronics and motor drives-An introduction and perspective," IEEE Transactions on Industrial Electronics, vol. 54, no. 1, pp. 14-33, 2007.

[14] T. Dragičević and M. Novak, "Weighting factor design in model predictive control of power electronic converters: An artificial neural network approach," IEEE Transactions on Industrial Electronics, vol. 66, no. 11, pp. 8870-8880, Nov. 2019.

[15] Y. Gao, T. Yang, X. Wang, S. Bozhko and P. Wheeler, "Machine Learning Based Correction Model in PMSM Power Loss Estimation for More-Electric Aircraft Applications," in 2020 23rd International Conference on Electrical Machines and Systems (ICEMS), Hamamatsu, Japan, November 2020, pp. 1940-1944. 